\title{
Simulation-based Design of Quartz Resonator for Bionic Olfactory Sensor
}

\author{
Meng Zhao, ${ }^{1 *}$ Jing $\mathrm{Ji}^{1},{ }^{1}$ and Toshitsugu $\mathrm{Ueda}^{2}$ \\ ${ }^{1}$ School of Electro-Mechanical Engineering, Xidian University, No. 2 South Taibai Road, Xi'an, 710071, P.R. China \\ ${ }^{2}$ IPS Research Center, Waseda University, 2-2 Hibikino, Wakamatsu-ku, Kitakyushu, Fukuoka 808-0135, Japan
}

(Received July 31, 2020; accepted January 28, 2021)

Keywords: bionic sensor, olfactory sensor, quartz resonator, simulation, anisotropic etching

In this paper, we report a bionic olfactory sensor that could be applied for breast cancer detection. This paper mainly focuses on describing the basic principle of the bionic olfactory sensor and introducing the simulation-based design of the sensor. We use an original anisotropic quartz etching simulation system to simulate the etching process of the key parts of the resonator, and thus obtain the key parameters of the quartz etching process of the resonator, so as to control the production accuracy of the quartz resonator and improve the performance of the bionic olfactory sensor.

\section{Introduction}

The early detection of cancer is important for treatment. The recurrence or metastasis of cancer is associated with the following factors: ${ }^{(1)}$ (1) the time from surgery to recurrence; (2) the site of metastasis; (3) age; (4) the response to prior treatment. Breast cancer is a malignant tumor occurring in the glandular epithelium of the breast. Among the people affected by breast cancer, $99 \%$ are women and only $1 \%$ are men, and breast cancer is a common threat to women's physical and mental health and a major public health problem. Since the 1990s, the global mortality rate of breast cancer has shown a downward trend. This is because of the development of breast cancer screening methods, resulting in an increased proportion of early detection, as well as the development of improved comprehensive treatment for breast cancer. Breast cancer has become one of the solid tumors most effectively treated. The examination methods for breast cancer are optic palpation, mammograms, breast ultrasound, fiber-optic duct scopes, cell aspiration diagnosis, needle biopsy, and surgical biopsy. However, most of these tests are time-consuming and expensive, and some tests are intrusive for patients. For example, a mammography for breast cancer can be embarrassing or painful when a special X-ray tool is clipped to the breast. In recent years, the breath and body odor of breast cancer patients have been analyzed by vapor chromatography. The odor associated with breast cancer is the so-called "pickle smell" or "strong onion smell". The results showed that the likelihood of producing these odorant molecules by symbiosis between cancer cells and microorganisms is very high. These

*Corresponding author: e-mail: mzhao@xidian.edu.cn

https://doi.org/10.18494/SAM.2021.3010 
odorants are detectable, and the design of olfactory sensors using a quartz crystal microbalance (QCM) is in progress. If breast cancer odor sensors are developed, they could shorten the time it takes to get a breast cancer test and provide a cheap and painless test.

Quartz has very stable physical and chemical properties, with little change in properties over a time scale of years. Resonators and oscillators require good frequency and temperature characteristics. Quartz crystal has been widely used in resonators and oscillators for a long time. This is because its temperature stability is high, the frequency changes are very small, and the performance is stable. A QCM with an adsorbed film superimposed on the quartz resonator layer by various chemical treatments can selectively detect target substances, and a wide variety of properties can be expected depending on the nature of the adsorption film. Patients with breast cancer have a characteristic odor that emanates from the site of the lesion, and if the odor is sensed, it can help in the early detection of breast cancer. This paper describes the first step in the development of a breast cancer bionic smell sensor: the development of a sensor for detecting ammonia.

\section{QCM Used in Bionic Olfactory Sensor}

In this study, the quartz resonator had gold electrodes on both sides of an $80 \mathrm{MHz}$ thin quartz plate. If a high-frequency voltage is applied between the electrodes, sliding deformation can occur at the same frequency and ultrasonic vibration can be generated. When driven at the mechanical resonant frequency, which is determined by the size of the quartz plate, the vibration is amplified, so the resonance is used as the resonator. When a mass is loaded on the electrode of the quartz resonator, the mechanical properties of the quartz plate are affected, and the resonant frequency is reduced. This effect is called the additive mass effect. ${ }^{(2)}$ The Sauerbrey equation has been established between the mass of the attached object and the vibration frequency when the elastic properties of the coating on the electrode of the quartz resonator are close to those of the quartz resonator and when the coating is thin enough for the viscosity of the attachment to be ignored. ${ }^{(3)}$ Therefore, by measuring the resonant frequency of the quartz resonator, the mass of the adsorbed material on the surface can be found, and if the density of the adsorbed material is also known, its thickness can be calculated.

The biomimetic olfactory sensor involved in this work has an adsorption film that can selectively adsorb ammonia on the electrode of a quartz resonator. ${ }^{(4)}$ The adsorption film has a three-dimensional structure of polycyclic aromatic hydrocarbons, silica particles, and tetraphenylporphyn-tetrasulfonic acid (Tpps). Ammonia molecules are adsorbed on Tpps, and the ammonia concentration is determined from the decrease in the vibration frequency caused by the change in mass. The mean sensitivity, defined by the relative frequency change for $1 \mathrm{ppm}$ ammonia in the sensor, is $0.1 \mathrm{ppm}$. Figure 1 illustrates the above process.

\section{Design of Quartz Resonator Used in QCM}

The frequency reduction caused by the additional mass effect must be considered when determining the quartz resonator thickness. Therefore, Sauerbrey deformation was used to 


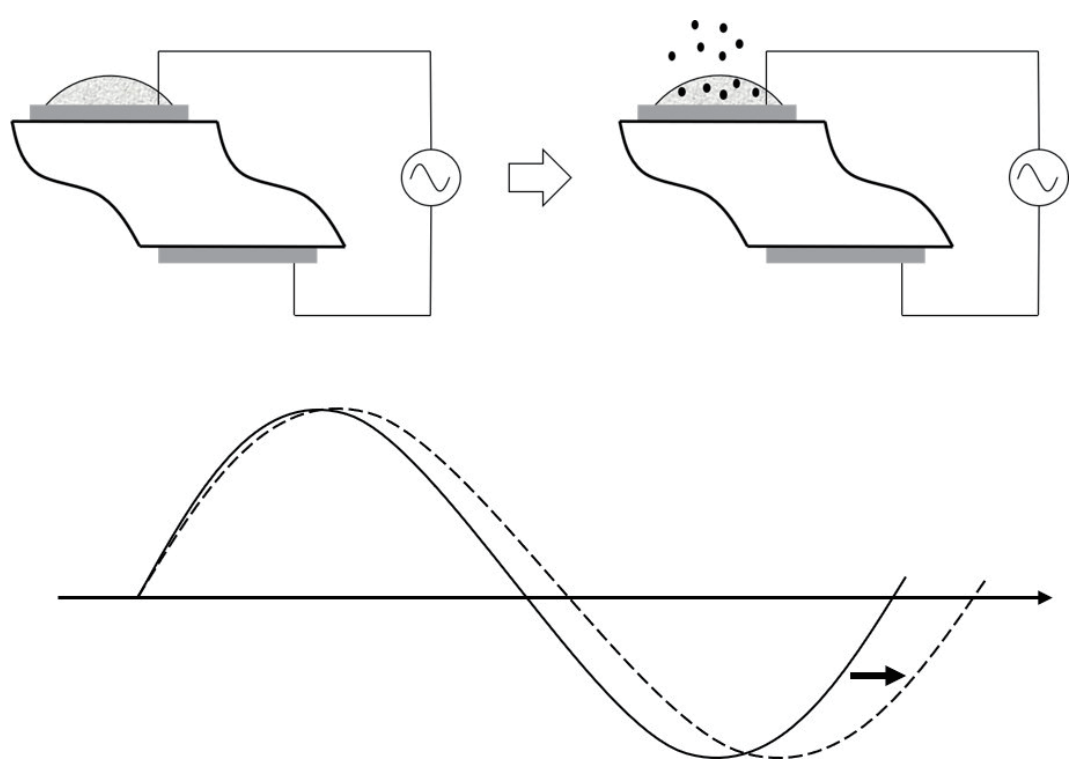

Fig. 1. An antigenic substance is fixed to the electrode of the quartz resonator, and if the odor molecules are attached to the antigenic substance, its vibration frequency changes.

determine the target frequency and the frequency reduction due to the added mass effect. We found that the quartz thickness was $10.24 \mu \mathrm{m}$; then, we determined the size of the quartz in the $X$ direction by reference to the result of vibration analysis using the finite element method (FEM). ${ }^{(5)}$ According to the FEM analysis results, to avoid spurious vibration coupling and achieve a good energy-trapping effect, the $X$-axis range should avoid values of 1330 and $1490 \mu \mathrm{m}$, and the range of $1350-1450 \mu \mathrm{m}$ is the best choice. Then, the electrode parameters were studied. The electrode structure of the quartz resonator was roughly divided into a full electrode and partial electrodes. The electrode that covers the entire two sides of the quartz resonator is called the universal electrode. Conversely, the electrodes that have a smaller area than the quartz plate are called partial electrodes. In the case of a full electrode on one side and a partial electrode on the other, the thickness of the quartz was sufficiently small relative to the width of the partial electrode, so that the power lines were parallel and could be approximately regarded as a partial electrode. A high quality factor (Q-factor) can be realized because the energy sealing effect can reduce energy loss and spurious vibration. ${ }^{(6)}$ Therefore, the current quartz resonator mainly used partial electrodes. Through the relationship between electrode size and energy limit analyzed by FEM, it was found that when an electrode with a length of 900 to $1400 \mu \mathrm{m}$ was installed on a piece of quartz with a length of $1400 \mu \mathrm{m}$, the analysis results showed that the smaller the size of the electrode, the smaller the spurious vibration coupling will be. However, if the electrode area is too small, the equivalent inductance value is too large and therefore not suitable for oscillating circuits. On the basis of the above judgment, the electrode size was finally set at $1000 \mu \mathrm{m}$. The final design of the resonator is shown in Fig. 2. 


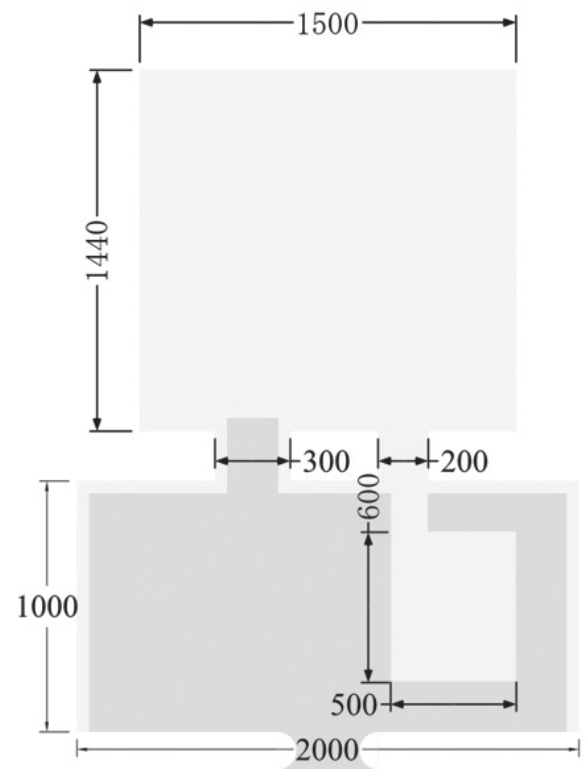

(a)

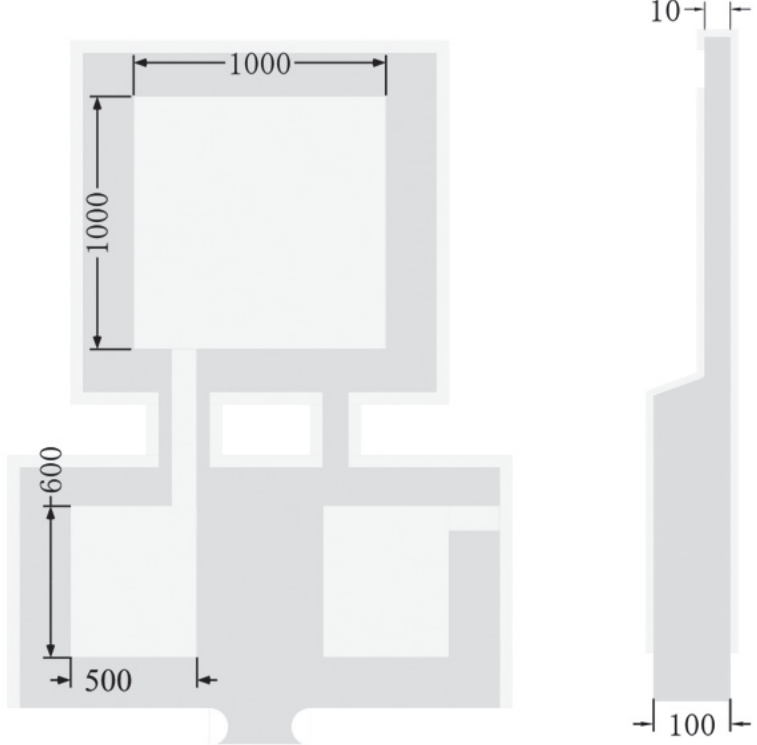

(b)

Fig. 2. Design of the quartz resonator (the lighter gray represents the electrodes). (a) Top view. (b) Bottom view (left) and section view (right).

\section{Processing Technology of Quartz Resonator}

The resonant frequency is determined by the thickness of the quartz, so it can be considered that the etching of the quartz is the most important part of the fabrication process. Etching can be roughly divided into chemical etching using a chemical reaction and physical etching using accelerated ions and lasers to impact on objects. The former is also called wet etching because it is carried out in an etching solution, and the latter is also known as dry etching because it occurs in an inert or reactive gas. Dry etching is good for deep vertical etching and can form precise patterns, but the etching device is bulky and expensive. Wet etching, on the other hand, can be performed with only beakers, which is economically advantageous. In addition, since the wet etching rate is more than 10 times that of dry etching, the processing time is also shorter. Wet etching can be divided into two types: wet etching of isotropic substances and wet etching using the anisotropic properties of crystals. V slots with a high aspect ratio and vertical sections can be easily machined in anisotropic etching. The reason for the anisotropic character of quartz is related to the density of the atomic arrangement. A surface with a low density, such as the $Z$ surface, has a high etching rate, while a surface with a high density, such as the $Y$ surface, has a low etching rate. For the same direction of quartz, if the temperature of the etching solution rises, the reactive energy increases, the chemical reaction rate on the quartz surface increases, and the etching rate increases. In addition, substances formed by chemical reactions in wet etching are transported through concentration diffusion. The etching rate is limited by the slow reaction and transport rates. If the reaction rate is limited, the spatial differences in etching rates 
caused by uneven spatial temperature can be reduced by improving the temperature distribution. ${ }^{(7)}$ In this work, the quartz was etched using ammonium difluoride aqueous solution as the etching solution. ${ }^{(8)}$

The fabrication process of the quartz resonator basically consists of two applications of lithography and two applications of spraying and wet etching of the quartz.

Preparation method of quartz etching solution:

(1) Stir the etching liquid while it is heated in a stirred water bath at $84 \pm 1{ }^{\circ} \mathrm{C}$.

(2) Confirm that the etching solution surface has formed a supersaturated film.

(3) Set the wafer on the fixture and place it in the etching solution.

(4) Soak the wafer for the necessary etching time.

(5) Rinse the wafer and heat the water bath to $80^{\circ} \mathrm{C}$.

(6) Rinse for 5 min twice, then rinse with running water in the beaker. Allow to dry naturally.

Preparation method of saturated aqueous solution of $\mathrm{NH}_{4} \mathrm{HF}_{2}$ :

(1) Add $600 \mathrm{cc}$ of ultrapure water to the beaker.

(2) Heat in a water bath.

(3) Weigh $50 \mathrm{~g}$ of ammonium difluoride powder using an electronic balance.

(4) Add the powder until saturation at the set temperature.

Saturated aqueous solutions of $\mathrm{NH}_{4} \mathrm{HF}_{2}$ are very dangerous, and corrosion-resistant aprons, gloves, and masks must be worn.

The main reasons for the variation of the etching rate are differences in the concentration and temperature of the etching solution. Concentration measurement and concentration control in etching are difficult, so the concentration is often set to be saturated. Saturation is controlled by the supersaturation of a thin film on the surface of the etching solution. The following experiments were conducted to investigate the relationship between temperature and etching rate. The temperature was changed from 81 to $85{ }^{\circ} \mathrm{C}$ in $1{ }^{\circ} \mathrm{C}$ steps, and wafers were etched for $100 \mathrm{~min}$. After that, the etching depth was measured and the etching rate was calculated. The results showed that there was a linear relationship between etching rate and temperature. The higher the etching rate, the smoother the surface, so a higher temperature gave better results. ${ }^{(8)}$ When wafers were etched at lower temperatures, colored streaks sometimes occurred on the surface. In addition, when the etching time was too long, the photoresist was damaged, so a higher etching rate was better. However, when the temperature was too high, the water evaporation rate increased and there was a danger of increased etching bias. In addition, it was difficult to keep the temperature stable. On the basis of the above comprehensive judgment, the etching temperature was finally selected as $84^{\circ} \mathrm{C}$.

\section{Simulation Design of Quartz Resonator}

The quartz resonator in this work was composed of three parts: the vibration part, the supporting part, and the fixed part. The parts related to the spurious vibration coupling are the vibration part and the supporting part. The fixed part is treated as a complete vibration isolator. To analyze the vibration part, an FEM vibration analysis method has been proposed, ${ }^{(9)}$ but the 
support part has not been fully analyzed. ${ }^{(10-12)}$ The etching of the quartz played an important role in determining the accuracy of the finished product. As shown in Fig. 2, the side view of the beam arm was designed to have a very large thickness difference, with the thickness changing greatly from one side to the other. By taking advantage of the anisotropic etching rate of the quartz in this direction, the whole shape with the varying thickness was formed simultaneously. The shape after etching, etching time, and solution temperature were predicted using the simulation system, whose graphical user interface (GUI) is shown in Fig. 3. ${ }^{(13-15)}$ We inputted the wafer type, wafer parameters, mask direction, mask size, and time step of the quartz, and then started the simulation. We obtained the simulation results for different etching times. After several applications of the infinite approximation method, we found that the etching shape was closest to our design size when the etching time was $69.8 \mathrm{~min}$ and the solution temperature was $84^{\circ} \mathrm{C}$. When we investigated the simulation results of different etching times in detail (Fig. 4), we found that the number of side wall faces does not change in the entire etching process, but the corrosion depth increases linearly with time, and the corrosion depth is determined by the $Z$ plane with the fastest etching rate. At about $80 \mathrm{~min}$, the crystal face was etched through, which should be avoided.

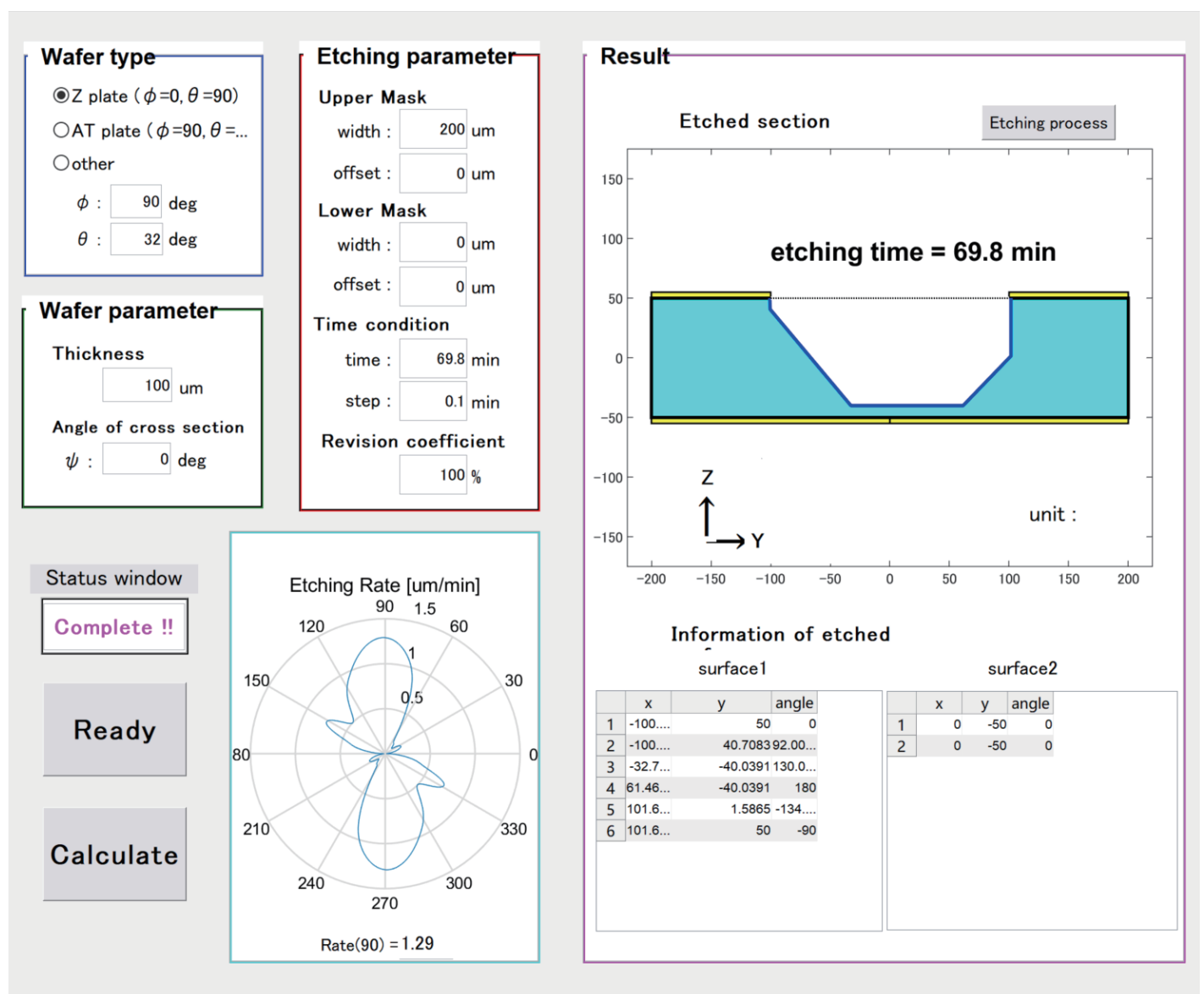

Fig. 3. (Color online) Simulator GUI and simulation result. 


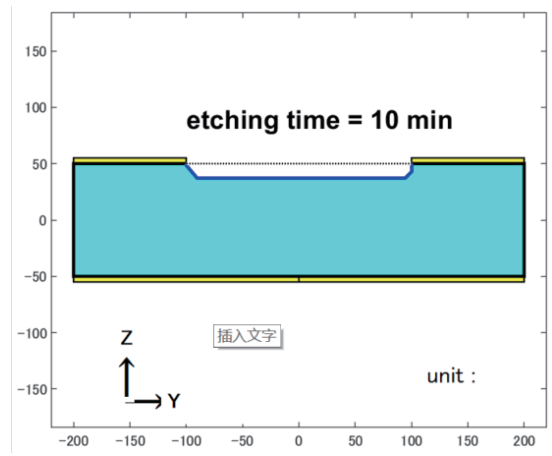

(a)

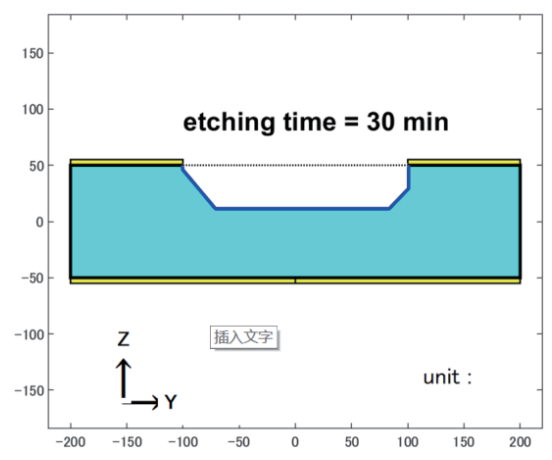

(c)

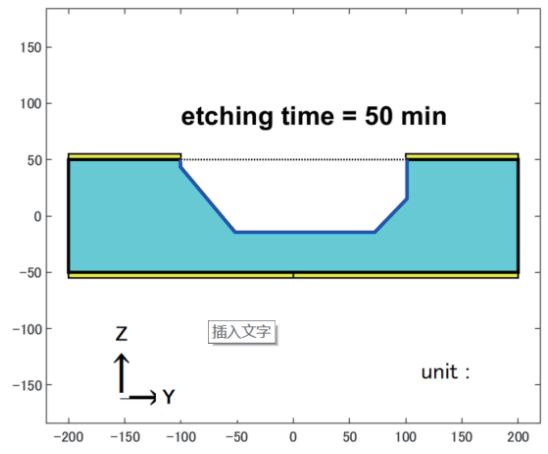

(e)

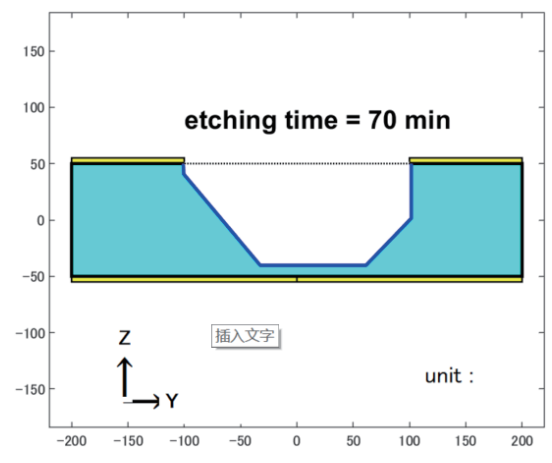

(g)

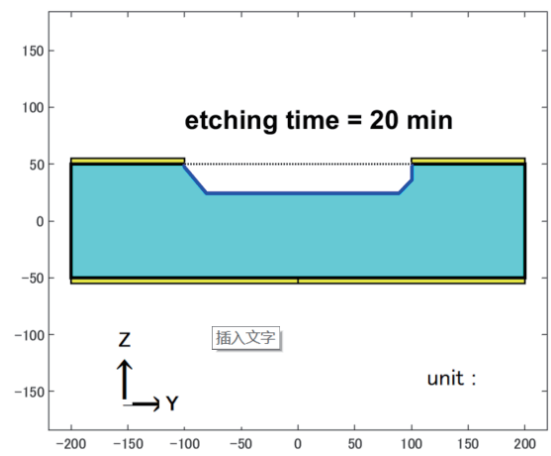

(b)

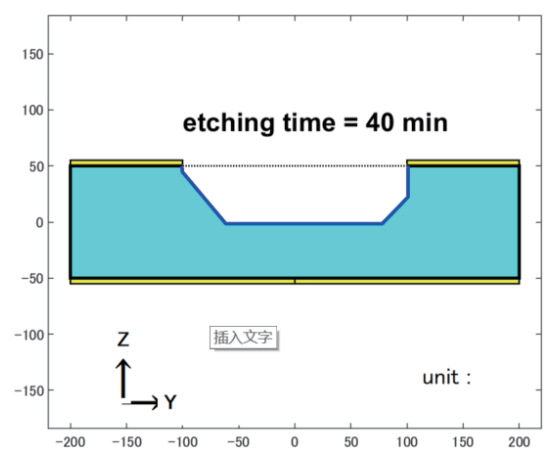

(d)

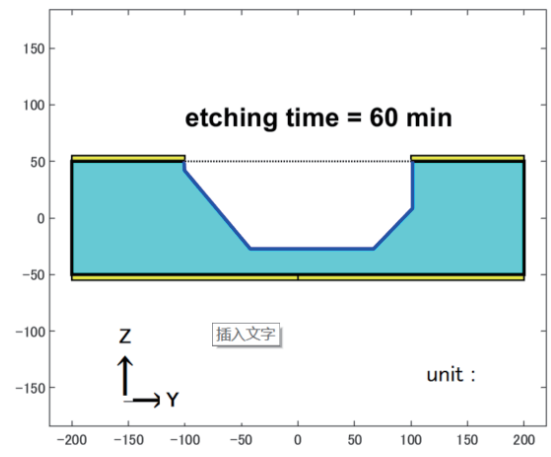

(f)

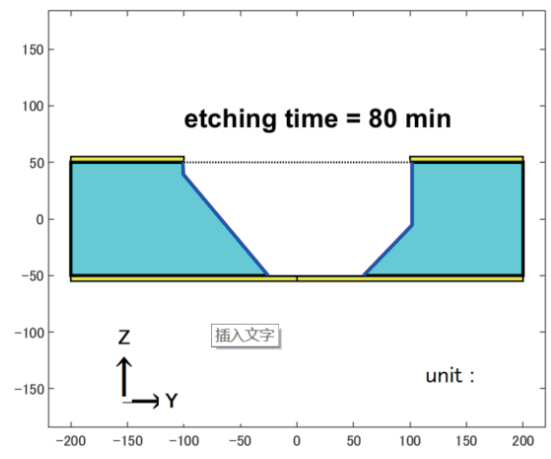

(h)

Fig. 4. (Color online) Simulation results for various etching times. (a) 10, (b) 20, (c) 30, (d) 40, (e) 50, (f) 60, (g) 70 , and (h) $80 \mathrm{~min}$. 


\section{Conclusions}

In this paper, we reported a bionic olfactory sensor for breast cancer detection. Its main principle is that it measures the frequency change of a quartz resonator caused by the change in the mass of adsorbate on the electrode to achieve the function of detecting trace odor markers of breast cancer. The main design parameters and key process steps of the sensor quartz resonator were introduced. We used an etching process simulation system of anisotropic quartz etching to simulate the key components of the resonator and thus obtain the key parameters of the etching process of the quartz resonator. In future work, we will optimize the three-dimensional simulation system to further improve the simulation results, so as to solve the problem of the irregular shape of some special edges and corners in the design process of quartz elements.

\section{Acknowledgments}

This work was supported by the National Natural Science Foundation of China (Grant No. 51702251).

\section{References}

1 M. D. Abeloff and H. Furue: Problems with Cancer and Its Treatment (Churchill Communications Japan, Tokyo, 1997).

2 T. Moriizumi: IEEJ J. 128 (2008) 370. https://doi.org/10.1541/ieejjournal.128.370

3 G. Sauerbery: Z. Physik 155 (1959) 206. https://doi.org/10.1007/BF01337937

4 K. Masashi and S. Seimei: Tech. Rep. IEICE, OME 100 (2000) 29. https://ci.nii.ac.jp/ naid/110003301250/

5 Y. Mizumachi: Record of 2006 Joint Conf. Electrical and Electronics Engineers in Kyushu (2012) 56.

6 K. Tan, J. Liang, and T. Ueda: 2008 IEEJ National Convention Proc. (IEEJ, 2008) 192.

7 K. Takai: Hitachi Chem. Tech. Rep. 52 (2009) 17. https://ci.nii.ac.jp/naid/10028107690/

8 T. Watanabe: Tech. Rep. IEICE, US 100 (2000) 23. https://ci.nii.ac.jp/naid/110003188343/

9 J. Ji, H. Oigawa, H. Chen, M. Zhao, and T. Ueda: IEEJ Trans. Sens. Micromach. 132 (2012) 275. https://doi. org/10.1541/ieejsmas.132.275

10 T. Ueda, F. Kohsaka, T. Iino, and D. Yamazaki: Trans. Soc. Instrument Control Eng. 23 (1987) 1233. https:// doi.org/10.9746/sicetr1965.23.1233

11 K. Hirama and Y. Aoyama: Tech. Rep. IEICE, US 96 (1996) 9. https://ci.nii.ac.jp/naid/110003187871/

12 H. Ueda and S. Watanabe: Tech. Rep. IEICE, US 97 (1997) 1. https://ci.nii.ac.jp/naid/110003187707/

13 M. Zhao, H. Oigawa, J. Wang, J. Ji, and T. Ueda: 2011 16th Int. Solid-State Sensors, Actuators and Microsystems Conf. (IEEE, 2011) 450-453.

14 M. Zhao, H. Ohigawa, J. Ji, and T. Ueda: Proc. IEEE Sensors (2011) 1693-1696.

15 M. Zhao and J. Ji: Sens. Mater. 30 (2018) 1053. https://doi.org/10.18494/SAM.2018.1833 


\section{About the Authors}

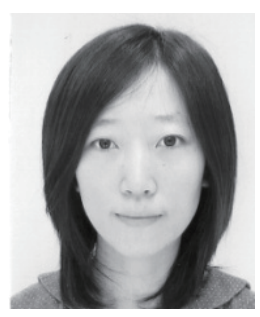

Meng Zhao received her B.Sc. degree in physics from Nankai University, China, in 2003 and her Ph.D. degree in astrophysics from Graduate University of the Chinese Academy of Sciences in 2009. From 2010 to 2015, she worked as a research associate and researcher in Information, Production and Systems Research Center, Waseda University, Japan. Since 2016, she has been an associate professor at Xidian University, P.R. China. She is currently engaged in the research and development of an anisotropic wet etching simulator of quartz.

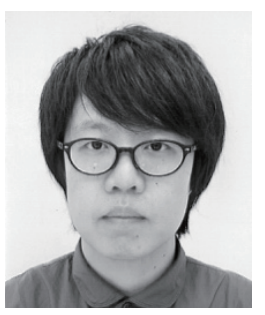

Jing Ji received his B.Sc. degree in microelectronic engineering from Nankai University, China, in 2003 and his M.S. and Ph.D. degrees in engineering of information, production, and systems from the Graduate School of Information, Production, and Systems, Waseda University, Japan in 2007 and 2013, respectively. From 2013 to 2016, he worked as a research associate and researcher in Information, Production and Systems Research Center, Waseda University. Since 2016, he has been an associate professor at Xidian University, P.R. China. He is a member of the Institute of Electrical and Electronics Engineers (IEEE), the Institute of Electrical Engineers of Japan (IEEJ), and the Japan Society of Applied Physics (JSAP). His research areas include quartz MEMS devices, the finite element method, and sensing technology.

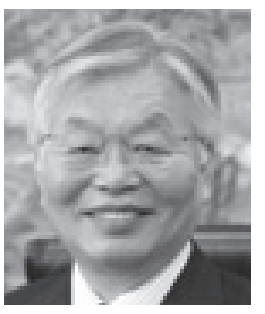

Toshitsugu Ueda received his B.E. and M.E. degrees in electrical engineering from Shinshu University, Nagano, Japan, in 1969 and 1971, respectively. He received his Ph.D. degree from Tokyo Institute of Technology in 1988. Since joining Yokogawa Electric Corporation in 1971, he has been engaged in developing low-noise amplifiers, mechanical resonators, micromachining technologies, and sensors using these technologies to control temperature, pressure, and displacement. He became a professor of the Graduate School of Information, Production, and Systems (IPS), Waseda University, in 2003 and dean of IPS and IPS Center in 2012. He received awards from the Society of Instrument and Control Engineers (Japan) in 1987 and 1994 and from the Japan Institute of Invention and Innovation in 1985 and 1987. He is a fellow of the Society of Instrument and Control Engineers (Japan) and a member of the Institute of Electrical Engineers (Japan) and the Society of Instrument and Control Engineers (Japan). 